\title{
On the Current System Design Examination and Path Reconstruction of Physical Education in China
}

\author{
Gong Tengyun \\ Nantong University, Nantong, China
}

\begin{abstract}
With the continuous advance of quality education system, teachers should pay more attention to the overall teaching effect in the process of teaching. In the course of physical education, the teachers should establish a more effective teacher-student interaction with students, so as to better stimulate the students' participation in learning and promote students to be engaged in sports learning actively. As to current physical education system in China, it requires teachers to explore earnestly both in the design ideas and teaching path, and look for the development mechanism which is more suitable to the era in practice. The paper makes a brief analysis of the significance of the current system design of physical education in China and also focuses on the interpretation of the current system design examination and path reconstruction of physical education, aiming to better boost the vigorous development of physical education in China, and provide effective teaching reference for teachers.
\end{abstract}

Keywords: physical education, current system, design, path

\section{The Significance of Current System Design of Physical Education}

\section{The Connotation of Current System Design of Physical Education}

In the process of actual teaching, only by ensuring the perfect and standardized operation of basic system can the optimizing operation of the processing mechanism of the whole project be ensured. As to the management system, it is not only a kind of control activity, but also the effective regulation of human behaviors. In the physical education system, in order to establish effective management measures, it is necessary to ensure the effective management and guidance to the behaviors of educators and educated personnel. Thus, the normalization and standardization of behaviors can be achieved. In the practical activity of actual management, both the management behaviors and managed behaviors should comply with the relevant standards, and at the same time, the role of system in controlling and regulating should also be improved (Du \& Zhang, 2014).

\section{The Era Necessity of the Current System Design of Physical Education}

In order to further promote the development of quality education, in the actual operating process of teaching behaviors, the establishment of effective system of physical education can better promote the behaviors of students and teachers. More and more attentions of the society have been paid to the health and physical problems of the adolescents. While, as the only way to enhance the physical fitness and training, physical education must also ensure the effective implementation and operation of corresponding regulations and norms, thus achieving the basic teaching goal of school education in a real sense. In addition, in the process

Gong Tengyun, Master, lecture, School of Sport Science, Nantong University. 
of proposing and establishing the Opinions on Strengthening the Sports and Physique of Adolescents, not only the teaching effect of moral education and aesthetic education should be optimized collectively, but also the optimized development of physical and intellectual education should be strengthened. Only in this way can the comprehensive development of talents be improved in the true sense (Zhong, Zhang, \& Wang, 2013), which puts forward the countermeasure and the suggestion from the aspects of society, school, family, and students. It also shows that China emphasis on youth sports work, specification and requirement from system.

\section{The Realistic Feasibility of the Current System Design of Physical Education}

The establishment of any system must make sure that it meets the basic feasibility in the actual operating process of the system. The system of physical education targets at catering to the interests and demands of the public. As a result, only by ensuring the effective safeguard of the public interests, can the system further obtain the support and recognition of public. In the actual operating process of the system, the current physical education not only meets the national interests, but also is in line with the requirements of personal development, which takes the sound development as the basic opportunity, and optimizes the comprehensive quality and ability collectively (Zheng \& Shao, 2014). That is to say, the introduction of the system is to further optimize the healthy image of China, promote the overall institutional environment and talent development requirement in China. It is also to realize the mature development of the social environment, and ensure the growth of economy and the progress of the society, which are all based on the quality upgrade of the talent. At the time of fully practicing the sports value and function, the optimized development of the whole social environment should be strengthened as well. In addition, the quality upgrade is the society's demand for talents in the 21 st century, so the physical education system also has the corresponding feasibility.

\section{The Analysis of the Current System Design of Physical Education}

\section{The Mechanism Problems of the Current System Design of Physical Education}

In the current system of physical education in China, there also exist the corresponding problems. The basic goal of the physical education is that teachers should make use of the effective teaching methods to promote the physical and mental development of students effectively. While they are enhancing the physical fitness of the students, they should also establish the optimized moral and quality education mechanism, promote the students to better finish the learning task to a certain degree and establish the optimized learning consciousness, thus better serving the socialist construction (Cheng \& Liu, 2013). In the process of practical management, the educational structure and educational system must ensure the optimization of overall teaching quality and the project; promote the close connection of educational activities and educational importance. In the actual project management, teachers should fully practice the design ideas and development requirements of the current system of physical education, and further optimize and operate of the important value of physical education. However, in the actual operation process of education system, there are still some prominent problems, and the corresponding national policy and the national system form the scientific design and project management. In order to achieve the system reform of the overall physical education, it is necessary to ensure the practical actions of reform, so as to meet the corresponding educational standards, and to avoid the fragmentation of the whole education mechanism and reform policy, otherwise, there will be educational plight, and even lead to the appearance of serious problems of the overall education mechanism in the process of implementation (Wang \& Fan, 2013). 


\section{The Objective Problem of the Current System Design of Physical Education}

The objective of the current system design of physical education is to ensure the positive achievement and profound implementation of the physical education, and too guarantee the physical health of adolescents fundamentally. But in the process of actual management, because the government at all levels and departments in charge of education still have some docking problems in the mechanism and in the design process, thus there exist some corresponding problems in the realization and implementation of the overall ultimate goal. If there is no clear development goal, it will be impossible to regulate and control the complete establishment of the overall structure of the physical education in an effective way, which will lead to the ineffective participation of students and teachers in it. In the actual management process, the corresponding management staff must address specific issues, so as to promote the effectiveness of the overall system in an effective way.

\section{The Evaluation Problem of the Current System Design of Physical Education}

In the current system of physical education, there are no effective education evaluation criteria. As a result, the corresponding educational outcomes can not be put into the effective practice as well. In the process of actual project processing, it will lead to the failure to establish the effective interaction and communication between the implementation and beneficiaries of physical education, and to effectively promote and evaluate the corresponding teaching behaviors. In the process of the whole project processing, it will impede the effective practice of the overall education and produce constraints in the actual process of establishing communication of education instructions and tasks. In particular, many schools do not put sufficient emphasis on the physical education, so the appraisal system of the whole educational structure and educational project is rather superficial, which not only restricts the overall education behaviors, but also hinders the effective realization of the corresponding self value (Hong \& Chen, 2015).

\section{The Auditing of the Current System Design of Physical Education}

\section{The Influence of Ideas and Cultures on the Current System Design of Physical Education}

The biggest auditing factor in the current system design of physical education is that the whole structure will be influenced by the ideas and cultures. In the process of establishing the system in China, because the culture is the basic support, the corresponding processing mode can be established only by ensuring that the overall operation structure of the project is in line with the basic social standard in the actual process of establishing management system. In China, the physical education system is influenced by traditional ideas, thus producing the theory of "scholars are at the pinnacle of social ranking," which also impedes the development process of physical education in China to some extent, and causes the overall project greatly influenced by the society in the operation process. In addition, in the traditional ideology, it is generally believed that the personal value can only be further promoted by ensuring the value of the group, which has also led to the bottleneck of the individualized development of people to a certain degree (Bian, 2014).

\section{The Influence of Value Orientation on the Current System Design of Physical Education}

The education system is the integration of the educational institutions and educational standards, and it needs the appropriate management personnel and project personnel to establish effective teaching behaviors, which operates effective management measures to the overall educational system and guides the corresponding teaching behaviors. However, due to the long-term influence of the value orientation, it has resulted in the lack of the naturalism education structure. The educational system project of China lays more emphasis on that the 
education should be based on serving the society, and ultimately target at meeting the social needs, which should not only boost the rapid development of society, but also ensure the optimizing operation of social interests.

\section{The Influence of Management Mechanism on the Current System Design of Physical Education}

In the process of actual management, the current system design of physical education will be affected by the value orientation, which will result in the problems of the whole educational structure and educational benchmark. Under the traditional educational idea, the corresponding management mechanism will be affected by the examination oriented education. Teachers do not pay the corresponding attention to the quality improvement of physical education and the establishment of corresponding teaching behaviors has no basic effectiveness, which will lead to the failure to establish effective richness of the corresponding sports teaching tasks. In the actual teaching process, schools will be affected by the school enrollment rate, and quality education will become empty, so the absence of actual effect is also the main cause of the problem in the current system of physical education (Sui, 2015).

\section{The Reconstruction Measures of the Current System Path of Physical Education}

\section{The Establishment of a Positioning Control Mechanism}

In the remodeling process of the current system design of physical education, the relevant management personnel should conduct the analysis and control of the corresponding projects in accordance with the actual work procedure and code of conduct, so as to make sure that the current system design of physical education can conform to the property requirements of the corresponding groups. In the process of establishing the traditional system, it mainly adopts the top-down model, which can guarantee the effective safeguard of public interests to a certain extent. However, once the passive relationship is established, the corresponding problems will exist in the process of implementation of the system. The projects that designers think are feasible and consistent with the interests of the public in the design process do not always mean that they can meet the actual needs of the audiences of each system (Huang, 2014). Therefore, in the process of practical system development and design, the corresponding design staff should effectively figure out the actual demand of the audiences, identify the practical problems of system design and the interest relations between the audiences of the system optimally, and establish the development embryo of equilibrium state. In addition, in the actual running process, the system personnel should collect practical advice in an effective way, so as to build the communication and interaction between the system and the audiences, reach a consensus effectively and further optimize the implementation and promotion of the system.

\section{The Establishment of Goal Management and Control Mechanism}

In the practical process of establishing and perfecting the educational system, the management personnel should focus on setting up the optimized project processing mechanism, and ensure the establishment of an effective goal management. In the actual operation structure of the project, it is necessary to ensure the management project to take the goal as the basic orientation, take the audiences as the center of the overall management project and take the final results as the management standard, thus achieving the establishment of effective sub-goals of the project. As to the physical education system in China, in the practical management, educational workers should not only optimize the establishment of the main target, but also establish phased targets with practical significance in accordance with the actual situation and the clear training mechanism of 
physical quality, truly promoting the physical ability of students and the all-round development of talents. In particular, China Sports Bureau formulated the Twelfth Five-Year Plan of Youth Sport and other documents in 2011 to further optimize the overall development goals of physical education, focus on the implementation of the education essence and connotation of physical education, actively explore and give full play to the enthusiasm of human, and ensure the effective implementation and development of management mechanism (Cheng \& Liu, 2016). In addition, in the process of establishing corresponding teachings goals, the design personnel and management personnel should establish the targeted mechanism in line with specific problems, so as to ensure the education system to meet the needs of individual and effectively promote the establishment of educational structure and system.

\section{The Establishment of the Mechanism of Supervision and Control}

In the actual teaching process, teachers should establish an effective control mechanism according to the corresponding problems; bring the management control structure into full play actively. They should not only ensure that the corresponding decision on the project is previous to the project supervision, but also ensure the effective decision-making management is conducted in the process of project implementation. The managers should make analysis and manage effectively in line with the basic theory and feedback control mechanism, ensuring the integrity and smooth operation of the whole supervisory structure. In the process of current system remodeling of the physical education, only the establishment of effective consciousness and behavior education to the implementing measures and implementing party can ensure the integrity of the overall supervision and control mechanism. In addition, it is necessary to organize the corresponding management personnel to correct the responsibility questions collectively in the process, and build the effective experience mechanism targeting at corresponding educational mechanism, thus effectively improving the current situation of physical education.

\section{The Establishment of an Evaluation Control Mechanism}

In the practical reform path of education system, the relevant management personnel should carry on effective evaluation of the effectiveness of the system in accordance with the specific situation and resort to the corresponding assessment measures to promote the actual working efficiency of overall physical education workers. At the same time, the operation of effective evaluation mechanism can further inspire and mobilize their working enthusiasm, and the establishment of an effective evaluation mechanism can also promote teachers to adjust their teaching behaviors. In addition, in the process of the current system remodeling of physical education, the optimizing operation of evaluation mechanism can better promote the teachers to more clearly identify their own responsibilities and obligations of education, truly optimizing the effect of management system and ensuring the learning interaction between teachers and students. The system supervision of administrative department by people's governments at all levels should take the goal as the basic guidance, and in the process of setting up the corresponding self-evaluation and self-development, they should operate the overall management measures of physical teaching smoothly. For the management project of physical education system, the basic means of evaluation should not only focus on promoting the overall quality and teaching structure of the physical education personnel, but also on better promoting the schools and teachers to devote themselves to the implementation of the system.

\section{Conclusion}

In a word, in the actual teaching process, the teachers should actively broaden their teaching ideas, and 
establish the targeted teaching guidance, thus contributing to the sustainable development of physical education in China. The corresponding teaching management personnel should ensure the optimizing process of the overall teaching project, according to the actual situation of education in China, and actively promote the corresponding teaching system to meet the social demand and the transformation from the perceptual cognition to rational cognition. Moreover, they should further boost the efficient integration of inheritance and development of the overall sportsmanship. At the same time, they should create the optimal educational environment, actively advance the process of quality education, use the optimal path to guarantee the combination of traditional education and modern education, thus enhancing the spiral development of the whole educational structure and better assisting the optimization of quality education for teenagers.

\section{References}

Bian, H. B. (2014). The review and reshape of the path of the current system design of physical education in China. New Campus (The First Third of a Month), 29(8), 246.

Cheng, W. G., \& Liu, X. (2013). Review and reconstruction of the current system of physical education in China. Journal of Shenyang Sport University, 32(3), 1-6.

Cheng, W. G., \& Liu, X. (2016). The review on the cause of anomie of the value appeals of physical education in China. Journal of Shenyang Sport University, 35(1), 1-6 \& 12.

Du, F., \& Zhang, S. W. (2014). The review and consideration of sports culture construction in colleges and universities of China. Journal of Physical Culture, 28(7), 141-144.

Hong, W., \& Chen, S. (2015). The survey and shaping of socialist core values in physical education. Journal of Inner Mongolia Normal University (Education Science Edition), 28(6), 76-78.

Huang, Y. J. (2014). Review and practical thinking on the sports and physical education. Journal of Anhui Vocational College of Police Officers, 22(1), 89-91.

Sui, H. (2015). Examining and thinking of the curriculum system and the social needs of physical education in the applied college under the context of The One Belt and One Road. Journal of Kashi Teacher's College University, 36(6), 73-76.

Wang, A. P., \& Fan, H. B. (2013). On the comparative examination of the sports teaching mode of traditional colleges and universities and "Three Independence" (pp. 7-11). The collected papers of The 19th Session of Papers Report of the National Institute of Higher Medical Education Seminar in Physical Education.

Zhong, M. B., Zhang, X. L., \& Wang, Y. (2013). The examination of compulsory sports of school during the new period. Journal of physical Culture, 22(1), 94-98.

Zheng, X. C., \& Shao, B. (2014). The examination of the school physical teaching in China since the founding of new China. Journal of physical Culture, 23(12), 114-117. 